\title{
ORIGINAL ARTICLE \\ Compliance with clean intermittent catheterization in spinal cord injury patients: a long-term follow-up study
}

\author{
SI Afsar, OU Yemisci, SNS Cosar and N Cetin
}

Study design: Retrospective review of medical notes.

Objective: To evaluate spinal cord injury (SCI) patients' compliance with bladder emptying method at long-term period after discharge and determine the frequency of urinary tract infections (UTIs).

Setting: Inpatient rehabilitation unit of tertiary research hospital.

Methods: Bladder management method of 164 new spinal cord injured patients were noted at discharge from rehabilitation center and follow-up. Patients were questioned whether they continued the initial bladder emtying method at follow-up, reasons for discontinuation and the history of treated UTIs.

Results: The most common bladder management method at discharge from inpatient rehabilitation center was clean intermittent catheterization (CIC) $(63.4 \%)$. At follow-up $42 \%$ of the patients who used CIC changed their bladder emptying method. Rate of reverting to urethral indwelling catheter (IC) was $21.4 \%$. Reasons for the patients who switched to IC application were recurrent UTIs, incontinence, nephrolithiasis, dependence on care givers and urethral strictures. For all patients, the frequency of treated UTI in 1 year was $38.8 \%$. The number of UTIs were highest in patients using IC.

Conclusion: Many factors, including urological complications, patient's preference, living environment, life-style and level of injury should be considered in deciding the method of bladder management in $\mathrm{SCl}$ patients. The $\mathrm{CIC}$ is a reliable and effective method in selected $\mathrm{SCl}$ patients. Despite changes in bladder emptying method, $\mathrm{ClC}$ was the most preferred method at long-term follow-up. Education of patients on catheterization technique and periodic follow-up is necessary to maintain patient compliance.

Spinal Cord (2013) 51, 645-649; doi:10.1038/sc.2013.46; published online 11 June 2013

Keywords: spinal cord injury; neurophatic bladder; bladder management; clean intermittent catheterization

\section{INTRODUCTION}

Despite the decrease of mortalitiy due to urological complications in the last decades, the urinary tract problems, stands out as an important cause of morbidity in patients with spinal cord injury (SCI). The impaired bladder function incurs a risk of developing various urological complications, such as urinary tract infections (UTIs), nephrolitiasis, incontinence and impaired renal function. ${ }^{1}$ Bladder management is a crucial element in improved outcomes for individuals with SCI. The primary goal of bladder management in SCI patients is to achieve adequate bladder drainage, low-pressure urine storage and low-pressure voiding. ${ }^{2}$ As its introduction by Lapides, clean intermittent catheterization (CIC) is established as a safe, effective and convenient treatment modality in selected SCI patients. ${ }^{3}$ It has become the standard procedure for managing the neuropathic bladder of SCI patients. ${ }^{4}$ At admission to our hospital most of the patients have an urethral indwelling catheter (IC). After full discussion and with the agreement of the patient, our policy in spinal cord injured patients, who do not regain normal micturation, is to carry out long-term CIC if possible, enabling them to be continent and catheter free. However, at long time follow-up, we observed that some of the patients changed their bladder management method without any medical consultation.
The aims of this study were, to determine SCI patients' compliance with the method of bladder management they used at inpatient rehabilitation discharge, investigate the reasons for discontinuing the regimen and identify the frequency of symptomatic UTI with different bladder management methods.

\section{MATERIALS AND METHODS}

The medical records of 164 new SCI patients admitted to Baskent University Ayas Physical Medicine and Rehabilitation Center from 1995 to 2005 were reviewed. Patients with poor cognitive functions and under 18 were not included in the study. Age, gender, education level, SCI etiology, level of neurological injury, completeness/incompleteness of injury based on The American Spinal Injury Association (ASIA) Impairment Scale (AIS), time since injury, length of stay in the hospital during rehabilitation period, functional state of patient at admission and at the end of rehabilitation right before discharge by using Functional Independence Measurement (FIM) scores and bladder emptying method at discharge and at every follow-up were recorded. The FIM is a functional assessment tool commonly used in the inpatient rehabilitation setting. Eighteen different activities (13 motor, 5 cognitive) are scored on a scale of 1-7 with a score of 1 indicating complete dependence and 7 indicating complete independence. For each case, the total FIM score on admission and at discharge were recorded. ${ }^{5}$ According to their bladder emptying method at discharge the patients were divided into four goups: (1) IC group; (2) CIC group; (3) reflex voiding group (subjects who 
used suprapubic tapping or spontaneous bladder emptying) and (4) normal voiding group (subjects who reported no signs of neurojenic bladder dysfunction). The primary source of follow-up was the medical records of the patients. Furthermore, all the patients were contacted by telephone. The patients were questioned and the informations specifically recorded included, the bladder emptying method they used after discharge, if the patient discontinued CIC, the time they quit CIC and the reason for discontinuation, type of catheter used by patients who continued with CIC (nelaton/hydrophilic), whether the patient had difficulty in obtaining the catheter, and the frequency of symptomatic UTI treated with antibiotics within 1 year. Symptomatic UTI was defined as significant bacteriuria ( $>10^{4}$ colonyforming units per $\mathrm{ml}$ ) plus one or more clinical signs and symptoms such as fever, suprapubic or flank discomfort, bladder spasm, increased spasticity, for which no other etiological cause could be identified. ${ }^{6}$

The study was approved by the hospital's Ethics Commitee and carried out according to the institutional guidelines and the principles of the Declaration of Helsinki.

\section{Statistical analysis}

Statistical analyses were performed with SPSS software (Statistical Package for the Social Sciences, version 13.0, SPSS Inc., Chicago, IL, USA). Independent samples $t$-test was used to compare parametric variables with normal distributions. Mann-Whitney test was used for between group comparisons, $\chi^{2}$ test was used to evaluate categorical variables and Spearman test was used to analyse correlations. A $P$-value of $<0.05$ with $95 \%$ confidence level was considered statistically significant.

\section{RESULTS}

A total of 164 SCI patients' medical records were reviewed and all the patients were contacted by telephone at follow-up. Demographic and clinical characteristic of the patients are presented in Table 1. The etiology of lesion was traumatic in 139 (84.8\%) patients and

Table 1 Demographic and clinical characteristics of the patients

\begin{tabular}{|c|c|c|c|}
\hline & $n$ & Mean ( \pm s.d.) & Range \\
\hline Age (years) & & $40.7 \pm 15.7$ & $23-65$ \\
\hline Time since injury (days) & & $51.5 \pm 56.8$ & $5-292$ \\
\hline Rehabilitation length of stay (days) & & $96.3 \pm 42.5$ & $31-169$ \\
\hline Duration of follow-up (months) & & $53.9 \pm 27.67$ & $5-84$ \\
\hline \multicolumn{4}{|l|}{ Education level } \\
\hline Illiterate & $4(2.6 \%)$ & - & - \\
\hline Primary education & $95(55.9 \%)$ & - & - \\
\hline High school & $33(21.7 \%)$ & - & - \\
\hline University & $30(19.7 \%)$ & - & - \\
\hline \multicolumn{4}{|l|}{ Gender } \\
\hline Female & $62(37.8 \%)$ & - & - \\
\hline Male & $102(62.2 \%)$ & - & - \\
\hline \multicolumn{4}{|l|}{ Level of injury } \\
\hline Tetraplegia & $43(26.2 \%)$ & - & - \\
\hline Paraplegia & $87(53 \%)$ & - & - \\
\hline Conus-Cauda Equina Syndrome & $34(20.7 \%)$ & - & - \\
\hline \multicolumn{4}{|l|}{ AIS grade } \\
\hline AIS A & $106(64.6 \%)$ & - & - \\
\hline AIS B & $17(10.3 \%)$ & - & - \\
\hline AIS C & $21(12.8 \%)$ & - & - \\
\hline AIS D & $20(12.1 \%)$ & - & - \\
\hline
\end{tabular}

Abbreviation: AIS, American Spinal Injury Association Impairment Scale. non-traumatic in $25(15.2 \%)$ patients. On the basis of neurological levels, $43(26.2 \%)$ of the patients were tetraplegic (C4-T1), 87 (53\%) were paraplegic (T2-T12) and $34(20.7 \%)$ had conus-cauda equina (L1-S4) injury. The method of bladder management at discharge from the rehabilitation center, was CIC in $104(63.4 \%)$ patients, IC in 16 $(9.8 \%)$ patients, reflex voiding in $25(15.2 \%)$ patients and normal voiding in $19(11.6 \%)$ patients. At discharge, CIC was the most common badder emptying method $(63.4 \%)$ and the majority of the patients on CIC were AIS A.

A total of 164 patients were followed for 5-84 months (average $53.9 \pm 27.67)$. Follow-up data revealed significant rate of change in the bladder emptying method. The method of bladder management at follow-up was CIC in 60 (37.5\%) cases, IC in 21 (13.1\%) cases, reflex voiding in $57(35.6 \%)$ cases and normal voiding in $22(13.8 \%)$ cases. Demographic and clinical characteristics of the patients according to their bladder emptying method at discharge and follow-up are given in Table 2. At follow-up, 44 (42\%) of the 104 patients stopped using CIC. Of the 44 patients, 9 (21.4\%) reverted to IC, 31 (73.8\%) started reflex voiding and $2(4.8 \%)$ regained normal voiding. None of the patients in the reflex voiding group or in the indwelling group shifted to CIC at follow-up, on the other hand two of the patients on IC group shifted to reflex voiding and one patient regained normal voiding at follow-up. Four of the 164 patients died of non-urological conditions at follow-up and 2 of these patients were on CIC at discharge. Demographic and clinical characteristics of patients who continued to use CIC and who stopped CIC are shown in Table 3. All

Table 2 Bladder emptying method at discharge from the rehabilitation center and follow-up

\begin{tabular}{cccc}
\hline IC & Intermittent & Reflex & Normal \\
& catheter & voiding & voiding
\end{tabular}

At discharge

No. of patients

Females

Males

Tetraplegic

Paraplegic

Conus-Cauda Equina

Syndrome

AIS grade

AIS A

AIS B

AIS C

AIS D

At follow-up
No. of patients
Females
Males
Tetraplegic
Paraplegic
Conus-Cauda Equina
Syndrome
AIS grade
AIS A
AIS B
AIS C

$16(9.8 \%) \quad 104(63.4 \%) 25(15.2 \%)$

$10(62.5 \%) \quad 70(67.3 \%) \quad 15(60 \%) \quad 7(36.8 \%)$

$8(50 \%) \quad 16(15.4 \%) \quad 11(44 \%) \quad 8(42.1 \%)$

$7(43.8 \%) \quad 70(67.3 \%) \quad 7(28 \%) \quad 3(15.3 \%)$

$1(6.3 \%) \quad 18(17.3 \%) \quad 7(28 \%) \quad 8(42.1 \%)$

$11(68.8 \%) \quad 86(82.7 \%) \quad 2(8 \%)$

$1(6.2 \%) \quad 7(6.7 \%) \quad 8(32 \%)$

$2(12.5 \%) \quad 8(7.7 \%) \quad 10(40 \%)$

$2(12.5 \%) \quad 3(2.9 \%) \quad 5(20 \%)$

$5(23.8 \%)$

$60(37.5 \%) 57(35.6 \%)$

$20(33.3 \%) 23(40.4 \%)$

$40(66.7 \%) 34(59.6 \%)$

$8(38.1 \%) \quad 11(18.3 \%) \quad 13(22.8 \%)$

$11(52.4 \%) \quad 42(70 \%) \quad 27(47.4 \%)$

$7(11.7 \%) \quad 17$ ( $29.8 \%)$

$2(9.5 \%)$

$19(90.5 \%)$

$2(9.5 \%) \quad 1(1.7 \%) \quad 16(28.1 \%)$

0

0

$6(10.5 \%)-22(100 \%)$

$22(13.8 \%)$

$13(59.1 \%)$

$9(40.9 \%)$

$8(36.4 \%)$

$6(27.3 \%)$

$8(36.4 \%)$
Abbreviations: AIS, American Spinal Injury Association Impairment Scale; IC, indwelling catheter. 
of the nine patients who reverted from CIC to IC were males and AIS A. The quitting time of CIC was $2-36$ months (average 16.09 \pm 12.77 ). The reasons for changing the method were reported as; recurrent symptomatic UTIs, incontinence, nephrolithiasis, dependence on care givers and urethral strictures. The reasons and level of injuries of the patients who stopped using CIC are given in Table 4. Of the patients who continued to use CIC at follow-up, $32(53.3 \%)$ used polyviniyl chloride (PVC) catheter while 28 patients (46.7\%) used hydrophilic catheter. Difficulty in maintaining hydrophilic catheter was expressed by 14 patients who lived in rural areas far from the city centers where medical facilities and availability of materials were limited.

A weak correlation was found between catheter types and compliance but there was no significant difference in compliance between patients using PVC catheter or hydrophilic catheter. Long-term compliance was not correlated with neither age nor level of lesion. Factors affecting long-term compliance with CIC are given in Table 5.

The FIM scores at admission and discharge were significantly higher in the CIC group than the IC group $(P=0.005$ and $P=0.013$, respectively). However, there were no statistical significant difference in admission and discharge FIM scores between the patients who continued to use CIC or used IC after discharge from the hospital at follow-up $(P=0.074$ and $P=0.236)$ (Table 6). Similarly, there were

Table 3 Demographic and clinical characteristics of patients who remained with $\mathrm{CIC}$ and changed $\mathrm{CIC}$

\begin{tabular}{lcc}
\hline & $\begin{array}{c}\text { Patients who remained } \\
\text { with CIC n (\%) }\end{array}$ & $\begin{array}{c}\text { Patients who } \\
\text { changed CIC n (\%) }\end{array}$ \\
\hline Gender & $20(33.3 \%)$ & $13(29.5 \%)$ \\
Female & $40(66.7 \%)$ & $31(70.5 \%)$ \\
Male & & \\
Level of injury & $11(18.3 \%)$ & $6(13.6 \%)$ \\
Tetraplegia & $42(70 \%)$ & $27(61.4 \%)$ \\
Paraplegia & $7(11.7 \%)$ & $11(25 \%)$ \\
Conus-Cauda Equina Syndrome & & \\
AIS grade & $55(91.7 \%)$ & $30(68.2 \%)$ \\
AIS A & $4(6.7 \%)$ & $4(9.1 \%)$ \\
AIS B & $1(1.7 \%)$ & $6(13.6 \%)$ \\
AIS C & 0 & $4(9.1 \%)$ \\
AIS D & &
\end{tabular}

Abbreviations: CIC, clean intermittent catheterization; AIS, American Spinal Injury Association Impairment Scale.

Table 4 The reasons and level of injuries of the patients who stopped CIC

\begin{tabular}{|c|c|c|c|c|c|c|}
\hline Patients & Level & $\begin{array}{l}\text { Recurrent } \\
\text { infection }\end{array}$ & Incontinence & Nephrolithiasis & Independent & $\begin{array}{l}\text { Urethral } \\
\text { stricture }\end{array}$ \\
\hline 1 & T2 & - & - & - & + & - \\
\hline 2 & C6 & - & - & - & - & + \\
\hline 3 & T3 & - & + & - & - & - \\
\hline 4 & $\mathrm{~T} 8$ & + & - & + & - & - \\
\hline 5 & $\mathrm{~T} 5$ & + & - & - & - & - \\
\hline 6 & $\mathrm{C} 4$ & + & + & - & - & - \\
\hline 7 & T12 & + & - & - & - & - \\
\hline 8 & T6 & - & + & - & - & - \\
\hline 9 & L1 & + & - & + & - & - \\
\hline
\end{tabular}

Abbreviation: CIC, clean intermittent catheterization. no significant difference in FIM scores of the patients who remained with $\mathrm{CIC}$ and those who changed (Table 7).

When all the patients where questioned, the frequency of UTI once a year was $38.8 \%$, two times per year $25.9 \%$, three times per year $30.6 \%$, four times per year $8.2 \%$, six times per year $2.4 \%$ and eight times per year was $1.2 \%$. The UTI frequency according to different bladder management methods have been given in Table 8 . There was no statistically significant difference in the frequency of UTI between

\section{Table 5 Factors affecting compliance with $\mathrm{CIC}$ at follow-up}

\begin{tabular}{lc}
\hline & Compliance with CIC, $r$ \\
\hline Age & 0.050 \\
Gender & -0.103 \\
Education level & -0.051 \\
Level of injury & 0.152 \\
AIS grade (discharge) & -0.115 \\
Catheter type & $0.448^{*}$ \\
Hydrophilic & $0.298^{*}$ \\
PVC & $0.275^{*}$
\end{tabular}

Abbreviations: AIS, American Spinal Injury Association Impairment Scale; CIC, clean intermittent catheterization; PVC, polyviniyl chloride. ${ }^{*} P<0.001$.

Table 6 FIM scores of the $\mathrm{CIC}$ and IC groups

\begin{tabular}{|c|c|c|c|}
\hline & $\mathrm{CIC}$ & IC & $\mathrm{P}$ \\
\hline \multicolumn{4}{|c|}{ Patients who used CIC or IC at discharge } \\
\hline FIM admission & $61.99 \pm 12.81$ & $54.87 \pm 15.35$ & $0.005^{*}$ \\
\hline FIM discharge & $83.41 \pm 16.85$ & $71.31 \pm 18.29$ & $0.013^{*}$ \\
\hline \multicolumn{4}{|c|}{ Patients who used CIC or IC after discharge } \\
\hline FIM admission & $60.46 \pm 11.70$ & $57.04 \pm 14.48$ & 0.074 \\
\hline FIM discharge & $78.35 \pm 15.92$ & $73.57 \pm 19.01$ & 0.236 \\
\hline
\end{tabular}

Table 7 FIM scores of the patients who remained with CIC and those who changed

\begin{tabular}{lccc}
\hline & $\begin{array}{c}\text { Patients who remained } \\
\text { with CIC }\end{array}$ & $\begin{array}{c}\text { Patients who } \\
\text { changed CIC }\end{array}$ & P \\
\hline FIM admission & $63.96 \pm 14.89$ & $64.13 \pm 14.11$ & 0.694 \\
FIM discharge & $85.00 \pm 20.41$ & $90.36 \pm 15.34$ & 0.161
\end{tabular}

Abbreviations: FIM, functional independence measure; CIC, clean intermittent catheterization. Data are mean \pm s.d.

${ }^{*} P<0.05$.

Table 8 The symptomatic UTI frequency according to different bladder management methods

\begin{tabular}{lcc}
\hline & UTI frequency/year & $\%$ \\
\hline IC & 3 & 93.3 \\
Intermittent catheter & 2 & 67.6 \\
Reflex voiding & 1 & 4.2 \\
Normal voiding & 0 & 0 \\
\hline
\end{tabular}

Abbreviations: IC, indwelling catheter; UTI: urinary tract infection. 
the IC and the CIC group $\left(P=0.132, \mathrm{X}^{2}=2.268\right)$. Similarly, there was no significant difference in the frequency of UTI between PVC and hydrophilic catheter users in the CIC group $(P=0.499)$. Also no significant association was found between educational levels and the frequency of UTI in all the groups $(P=0.987)$.

\section{DISCUSSION}

In SCI patients where spontaneous voiding could not regained, CIC, is considered to be the most appropriate and safest bladder management method in terms of urologic complications. ${ }^{7}$ Our standard neuropathic bladder protocol in SCI patients is to perform urine analysis, creatinine clearance, ultrasound examinations and urodynamic studies during their stay and at follow-up if possible, initiate oral anticholinergic medication to patients with a reflex bladder and sustained high intravesical pressures and switch to CIC if possible. As soon as the patient's condition is stable oral fluid intake is restricted to 2000 meals per day and CIC 5 to 6 times per day is started. Those patients with inadequate hand function to selfcatheterise depend on family members or care attendants to carry out the technique. Review of the literature reveals high levels of changing methods from CIC to IC in SCI patients. Perkash et al. ${ }^{3}$ have followed 50 patients with an average of 22 months, and have reported that $66 \%$ of the patients stopped using CIC at follow-up. In a study by Dahlberg et al. ${ }^{8}$ the rate of use of CIC have been reported as $36 \%$ in 129 patients with 18 years of average duration of injury, however, they also reported that $65 \%$ of these subjects also used other methods for bladder emptying. Changes in bladder emptying methods in SCI patients partially depend on newly emerging informations in the literature. With time, bladder management with CIC has increased in popularity. Cameron et al. ${ }^{9}$ have reported that the use of CIC increased from $12.6 \%$ in 1972 to a peak of $56.2 \%$ in 1991. However, only $20 \%$ of patients initially on CIC remained on this form of bladder management. They also reported that IC use initially decreased from $33.1 \%$ in 1972 to $16.5 \%$ in 1991 but increased to $23.1 \%$ in $2001 .{ }^{9}$ In our study, the rate of CIC use at discharge was $63.4 \%$, at follow-up, this rate decreased to $37.5 \%$ while the rate of reflex voiding increased from $15.2 \%$ to $35.6 \%$. However, we found that the most common used method of bladder emptying at followup was still CIC.

Studies have suggested that the rate of reverting to IC from CIC is higher in female patients and it has been reported that females find this procedure more distressing and uncomfortable than males. ${ }^{4,10}$ Timoney et al. ${ }^{11}$ have reported compliance with CIC as 50\% in female patients. The major reasons for low-compliance rates in these studies were the persistence of urinary incontinence despite anticholinergic therapy and lack of availability of external collective devices in female patients. ${ }^{4,11}$ In contrast to the literature, all of the nine patients who reverted from CIC to IC in our study were males. This may be explained by the fact that most of our female patients using CIC were paraplegic $(88.3 \%)$ cases who could perform catheterization independently and that only four of them were tetraplegic.

Individuals with tetraplegia, regardless of gender, report greater satisfaction with suprapubic catheter or IC use compared with CIC. ${ }^{2}$ Similarly, in our study $50 \%$ of the tetraplegic patients were placed on IC. The reasons in two patients who changed their bladder emptying method from CIC to IC were inadequate hand function and lack of family support to carry out the technique.

On the other hand, we found that FIM scores at admission and discharge were significantly higher in the CIC group than the IC group, however, there were no statistical significant difference in FIM scores of the patients who remained with CIC and those who changed, which suggests that even though some patients were able to perform CIC they moved into the IC group. This shows that FIM scores does not have a significant role in compliance and other factors related with patients' adherence should be further investigated.

There are also several studies reporting higher long-term compliance with CIC. These studies revealed compliance rates ranging from 71 to $86 \%$ at $5-10$ years follow-up. ${ }^{12-15}$ In our study, compliance with CIC was $57.7 \%$, lower than those reported by other studies. Among patients who discontinued CIC, $73.8 \%$ started to have reflex voiding and $4.8 \%$ regained normal voiding. This may be due to high number of patients with AIS D in our study. Chai et al., ${ }^{12}$ Hill et al., ${ }^{13}$ Gallien et al., ${ }^{14}$ and Maynard et al. ${ }^{15}$ reported the rate of changing from CIC to IC as $6.7 \%, 15 \%, 6 \%$ and $6 \%$, respectively. Similarly, the rate of discontinuing CIC and changing to IC was $8.6 \%$ in our study.

Several factors including (1) persistent incontinence despite anticholinergic agents; (2) severe spastisity of lower extremities; (3) trauma to the lower urinary tract and orchiepididimitis; (4) severe upper urinary tract complications, such as pyelonephritis and hydronefrosis have have been found responsible for discontinuaton of CIC. ${ }^{16}$ In our study, the reasons for discontinuing $\mathrm{CIC}$ were recurrent symptomatic UTI, incontinence, nephrolithiasis, dependence on care givers and urethral stricture. Spasticity was not one of the reasons in our study because CIC was not preferred as a method of bladder drainage in patients with severe spasticity in the lower extremities despite medical therapy, at discharge. This might also explain why upper urinary tract complications such as hydronephrosis were not observed at follow-up in this study. On the contrary, as the subjects in this study were followed for a maximum of 7 years, it was possible that insufficient time had elapsed for different bladder management methods to cause significant changes in the upper urinary tract.

The overall rate of UTI in the literature changes from 1.82 to 2.5 episodes per year. ${ }^{17,18}$ In our study, most of our patients $(38.8 \%)$ reported UTI as once a year. Dahlberg et al. ${ }^{8}$ have reported that, the maximum frequency of UTI was seen in the group that combined CIC with other methods such as suprapubic tapping, spontaneous reflex voiding, compression or straining and UTI frequency was lower in the group using suprapubic tapping alone than the group using CIC alone. Similarly, in our study, the number of UTI was lower in the reflex voiding than the CIC group. Furthermore, in accordance with the literature the incidence of UTI was highest in the IC group. The reported benefits of hydrophilic catheters compared with PVC catheters include, reduced UTIs, reduced microhematuria and high levels of patient satisfaction. ${ }^{19}$ In a metaanalysis that examined six studies including 548 patients with SCI, during the implementation of CIC, the groups using hydrophilic catheter and nonhydrophilic catheter were compared and the incidence of UTI was found to be lower in the group using hydrophilic catheter. ${ }^{20}$ In our study, 32 (53.3\%) patients used PVC catheters and 28 (46.7\%) patients used hydrophilic catheter. The incidence of UTI was 2 episodes per year for PVC catheter users and once a year for hydrophilic catheter users. Although the difference between the two groups was not statistically significant, the subjects who used hydrophilic catheter had less UTI than those who used PVC catheter $(P=0.05)$.

Recurrent symptomatic UTIs can be a problem for many patients performing CIC at long-term. ${ }^{21}$ The causes of UTIs in patients performing $\mathrm{CIC}$ are inadequate frequency of emptying, inadequate emptying at the time of catheterization, poor catheterization technique and catheter care and traumatic catheterization. Bladder stones, caused by the introduction of pubic hair or loss of the catheter in the bladder, can also be seen in patients performing long-term CIC. ${ }^{22}$ Bladder stones may also be one of the causes of recurrent 
UTIs. Symptomatic UTIs have a negative impact on long-term compliance. In case of nephrolithiasis or UTI, oral hydration and pain management are a part of acute treatment. ${ }^{23}$ Most of the SCI patients performing CIC are placed with urethral IC in emergency departments or medical centers at the acute period of UTI or nephrolithiasis. However, at long-term period these patients aren't informed about removal of IC and continuing with CIC regimen. Recognition of appropriate removal of IC should be improved among general practitioners and residents.

In determinig compliance, first of all physicians needs to identify the barriers to implementing intermittent catheterization. ${ }^{24}$ Correct choice of catheter, which is most appropriate for the patient is necessary. Proper education of patients and urgent need for provision of trained care givers to perform CIC is essential. Furthermore, assessment of catheterization technique and professional instruction on catherization technique to avoid trauma to the urethra and periodic urologic follow-up is necessary to obtain and maintain patient compliance. Another important factor is the motivation of patients and care givers to carry out intermittent catheterization four to six times a day. In a recent study by Akkoc et al. ${ }^{25}$ it was reported that the patients experiencing the highest distress and limitations were those using CIC performed by a caregiver/attendant.

As the limitations of our study, although primary source of followup was medical records from outpatient clinics, all of the patients were contacted by phone and therefore urine cultures, ultrasound and urodynamic data could not be obtained in most patients at follow-up. Moreover, urinary complications were not addressed in this study.

\section{CONCLUSION}

This study highlights the significance of compliance rate evaluation, which is a very important variable to determine the treatment success and the health system efficiency. The optimal method of bladder management still remains controversial in SCI patients. Many factors including urological complications, patient's preference, living environment, life-style and level of injury should be considered in deciding the method of bladder management in SCI patients. We agree with the authors that inappropriate selection of bladder management method not only adversly affects a patient's quality of life, but also has a significant detrimental impact on the economic status of the health-care system. ${ }^{7}$

CIC is the most preferred, reliable and successful long-term bladder emptying method in selected SCI patients who can peform catheterization independently. Nevertheless, if there is sufficient family or care attendants support to carry out the technique, CIC should be preferred for bladder management even in patients with inadequate hand functions. However, periodic follow-up and effective education of the patients and caregiver/attendants on long-term CIC use, complications and catheter care are essential for long-term compliance.

\section{DATA ARCHIVING}

There were no data to deposit.

\section{CONFLICT OF INTEREST}

The authors declare no conflict of interest.

1 Hansen RB, Biering-Sørensen F, Kristensen JK. Bladder emptying over a period of 10-45 years after a traumatic spinal cord injury. Spinal Cord 2004; 42: 631-637.

2 Linsenmeyer TA, Bodner DR, Creasey GH, Green BG, Groah SL, Joseph A et al. Bladder management for adults with spinal cord injury: a clinical practice guideline for health care providers. J Spinal Cord Med 2006; 29: 527-573.

3 Perkash I, Giroux J. Clean intermittent catheterization in spinal cord injury patients: a follow up study. J Urol 1993; 149: 1068-1071.

4 Yavuzer G, Gök H, Tuncer S, Soygur T, Arikan N, Arasil T. Compliance with bladder management in spinal cord injury patients. Spinal Cord 2000; 38: 762-765.

5 Granger CV. Quality and outcome measures for medical rehabilitation. In Braddom RL (ed) Physical Medicine and Rehabilitation, 2nd edn. Saunders Elsevier: Philadelphia, pp 151-164, 2000.

6 Darouiche RO. Infections in patients with spinal cord injury. In: Mandell GL, Bennett $\mathrm{JE}$ and Dolin R eds. Principles and Practice of Infections Diseases, 6th edn. Elsevier Churchill Livingstone: Philadelphia, pp 3512-3517, 2005.

7 Weld KJ, Dmochowski RR. Effect of bladder management on urological complications in spinal cord injured patients. J Urol 2000; 163: 768-772.

8 Dahlberg A, Perttilä I, Wuokko E, Ala-Opas M. Bladder management in persons with spinal cord lesion. Spinal Cord 2004; 42: 694-698.

9 Cameron AP, Wallner PL, Tate DG. Bladder management after spinal cord injury in the United States 1972 to 2005. J Urol 2010; 184: 213-217.

$10 \mathrm{Ku}$ Ja Hyeon. The management of neurogenic bladder and quality of life in spinal cord injury. BJU Int 2006; 98: 739-745.

11 Timoney AG, Shaw PJR. Urological outcome in female patients with spinal cord injury: the effectiveness of intermittent catheterisation. Paraplegia 1990; 9: 556-563.

12 Chai T, Chung AK, Belville WD, Faerber GJ. Compliance and complications of clean intermittent catheterization in the spinal cord injured patient. Paraplegia 1995; 33: 161-163.

13 Hill VB, Davies WE. A swing to intermittent clean self catheterization as a preferred mode of management of the neuropathic bladder fort he dextrous spinal cord patient. Paraplegia 1988; 26: 405-412.

14 Maynard FM, Glass J. Management of the neuropathic bladder by clean intermittent catheterization: 5 year outcomes. Paraplegia 1987; 25: 106-110.

15 Gallien P, Nicolas B, Robineau S, Le Bot MP, Durufle A, Brissot R. Influence of urinary management on urologic complication in a cohort of spinal cord injury patients. Arch Phys Med Rehabil 1998; 79: 1206-1209.

16 Mizuno K, Tsuji T, Kimura A, Liu M, Masakado Y, Chino N. Twenty-seven years of complication-free life with clean intermittent self-catheterization in a patient with clean intermittent self-catheterization in patient with spinal cord injury: a case report. Arch Phys Med Rehabil 2004; 85: 1705-1707.

17 Siroky MB. Pathogenesis of bacteriuria and infection in the spinal cord injured patient. Am J Med 2002; 113: 67S-79S.

18 Waites KB, Canupp KC, DeVivo MJ. Epidemiology and risk factors for urinary tract infection following spinal cord injury. Arch Phys Med Rehabil 1993; 74: 691-695.

19 Chartier-Kastler E, Denys P. Intermittent catheterization with hydrodhilic catheters as a treatment of chronic neurogenic urinary retention. Neurourol Urodyn 2011; 30 : 21-31.

20 Li L, Ye W, Ruan H, Yang B, Zhang S. Impact of hydrophilic catheters on urinary tract infections in peoples with spinal cord injury: systematic review and meta-analysis of randomized controlled trials. Arch Phys Med Rehabil 2013; 94: 782-787.

21 Heard L, Buhrer R. How do we prevent UTI in people who perform intermittent catheterization? Rehabil Nurs 2005; 30: 44-45.

22 Newman DK, Willson MM. Review of intermittent catheterization and current best practices. Urol Nurs 2011; 31: 12-28.

23 Preminger GM, Tiselius HG, Assimos DG, Alken P, Buck AC, Gallucci M et al. EAU/AUA Nephrolithiasis Guideline Panel. 2007 guideline for the management of ureteral calculi. J Urol 2007; 178: 2418-2434.

24 Vaidyanathan S, Soni BM, Singh G, Oo T, Hughes PL. Barriers to implementing intermittent catheterisation in spinal cord injury patients in Northwest Regional Spinal Injuries Centre, Southport, U.K. ScientificWorldJournal 2011; 11: 77-85.

25 Akkoç Y, Ersöz M, Yıldız N, Erhan B, Alaca R, Gök H et al. for Neurogenic Bladder Turkish Research Group. Effects of different bladder management methods on the quality of life in patients with traumatic spinal cord injury. Spinal Cord 2013; 51: 226-231. 